\title{
Psychiatric and medical admissions observed among elderly patients with new-onset epilepsy
}

\author{
Laurel A Copeland ${ }^{1,2^{*}}$, Alan B Ettinger ${ }^{3}$, John E Zeber ${ }^{1,2}$, Jodi M Gonzalez ${ }^{4}$ and Mary Jo Pugh ${ }^{5,6}$
}

\begin{abstract}
Background: Inpatient utilization associated with incidence of geriatric new-onset epilepsy has not been characterized in any large study, despite recognized high levels of risk factors (comorbidity).
\end{abstract}

Methods: Retrospective study using administrative data (Oct '01-Sep '05) from the Veterans Health Administration from a nationwide sample of 824,483 patients over age 66 in the retrospective observational Treatment In Geriatric Epilepsy Research (TIGER) study. Psychiatric and medical hospital admissions were analyzed as a function of patient demographics, comorbid psychiatric, neurological, and other medical conditions, and new-onset epilepsy.

Results: Elderly patients experienced a 15\% hospitalization rate in FY00 overall, but the subset of new-onset epilepsy patients $(n=1,610)$ had a $52 \%$ hospitalization rate. New-onset epilepsy was associated with three-fold increased relative odds of psychiatric admission and nearly five-fold increased relative odds of medical admission. Among new-onset epilepsy patients, alcohol dependence was most strongly associated with psychiatric admission during the first year after epilepsy onset (odds ratio $=5.2 ; 95 \%$ confidence interval 2.6-10.0), while for medical admissions the strongest factor was myocardial infarction (odds ratio $=4.7 ; 95 \%$ confidence interval 2.7-8.3).

Conclusion: From the patient point of view, new-onset epilepsy was associated with an increased risk of medical admission as well as of psychiatric admission. From an analytic perspective, omitting epilepsy and other neurological conditions may lead to overestimation of the risk of admission attributable solely to psychiatric conditions. Finally, from a health systems perspective, the emerging picture of the epilepsy patient with considerable comorbidity and demand for healthcare resources may merit development of practice guidelines to improve coordinated delivery of care.

\section{Background}

Epilepsy is a common neurologic disorder among persons over age 65 , with a prevalence estimated at 5.7 cases per 1,000 older adults [1,2]. Incidence may range from 16 to 51 cases per 100,000 in population-based studies [3]. While the diagnosis and treatment of seizures in this population is a challenge in its own right, comorbid conditions may lead to hospital admissions for reasons that go beyond epilepsy. Comorbidities with elevated occurrence among epilepsy patients include digestive system disorders, stroke, respiratory disorders, dementia, migraine and other pain conditions as well as psychiatric disorders [3-5]. If the risk and nature of non-epilepsy hospitalizations are substantial, this means

\footnotetext{
* Correspondence: LaurelACopeland@gmail.com

'Veterans Affairs: Central Texas Veterans Health Care System (CAHR), 2102 Birdcreek Dr, Temple, TX 76502 USA

Full list of author information is available at the end of the article
}

that elderly new-onset epilepsy patients are a special population in whom clinicians should pay careful attention to identifying and addressing comorbidity [6]. In this context, epilepsy becomes a marker for a constellation of serious conditions that go substantially beyond the epilepsy itself. Yet little is known about the rate and nature of hospitalizations among elderly patients with new-onset epilepsy.

Previous research on older epilepsy patients treated in the VA noted a large burden of psychiatric disorders, [7] which may contribute directly to inpatient stays. Seizure disorders, other medical conditions including cerebrovascular disorders, [5] or psychiatric conditions may necessitate inpatient admission among elderly patients with epilepsy [8]. These inpatient experiences have rarely been described outside economic analyses and studies of emergency department use [9-11].

\section{Biomed Central}


As part of the Treatment In Geriatric Epilepsy Research (TIGER) study of new-onset late-life epilepsy, we examined risk factors for psychiatric and medical admissions among geriatric patients, comparing those with new-onset epilepsy to those without epilepsy, expecting that TIGER patients would have higher relative odds of admission. Next we analyzed admissions of new-onset epilepsy patients only, to better understand risk factors for medical and psychiatric hospitalization in that clinically unique subset of patients.

\section{Methods}

\section{Study design}

The TIGER study is a national retrospective, observational study of new-onset epilepsy in geriatric patients receiving care in the Veterans Health Administration (VA).

\section{Sample}

The sample consisted of VA patients aged 66 or older in fiscal year 2000 (FY2000; Oct 1999-Sep 2000). The prior two years were used to establish epilepsy status as no epilepsy, ongoing epilepsy, or new-onset epilepsy. Based on a validated algorithm comparing chart review data to administrative and Medicare ICD-9 codes and electronic medication records, both diagnosis of a seizure disorder (ICD-9 code 780.3 or 345) and a current anti-epileptic drug (AED's) prescription were required to establish epilepsy status. The algorithm has a positive predictive value of 0.98 [12]. New-onset epilepsy was defined by use of the VA without diagnosis of a seizure disorder in FY1998-FY1999, and diagnosis of seizure disorder plus receipt of AED's in FY2000 but not in FY1998-FY1999. Patients with previous diagnosis of epilepsy (prevalent cases) were excluded $(n=41,867)$. This study was approved by the Institutional Review Boards at the University of Texas Health Science Center at San Antonio and at the Bedford and Hines VA Medical Centers prior to initiation.

\section{Data sources}

Administrative data extracts of the VA's all-electronic medical record provided measures of diagnoses, inpatient and outpatient utilization, and demographics.

\section{Measures}

The years FY1998-FY1999 were also used to establish neurological conditions associated with seizure disorders present prior to determining epilepsy onset. These "prior" conditions included diagnosis of senile dementia per Krishnan, [13] cerebrovascular disorders (ICD-9 codes 430-438), brain tumor (ICD-9 191), head injury (ICD-9 851-854), and other neurological conditions such as multiple sclerosis and Parkinsons [14].
Then administrative data extracts determined hospitalizations during the first year (FY2000) and comorbid conditions, while the second year (FY2001) established ongoing use of the VA healthcare system (yes or no). Sociodemographic measures included age group (youngold 65-74 years of age, older 75-84, oldest 85+), ethnicity/race (African-American or Hispanic versus white), and gender. Patients missing data on race were identified and excluded (20\% of sample).

The predictors of interest were demographic measures, prior neurological conditions, other comorbid medical conditions, psychiatric conditions, and newonset epilepsy. Selim comorbidity indicators, developed on VA patient survey data and subsequently adapted to administrative data $[15,16]$ were employed to identify the comorbid medical and psychiatric conditions. The Selim indicators assess diagnoses for diabetes, hypertension, myocardial infarct, angina, arrhythmia, congestive heart failure, and transient ischemic attacks, among other conditions. The Selim indicator of seizures was not included in analytic models as these diagnosis codes were incorporated into the new-onset epilepsy indicator, leaving 29 Selim physical comorbidity indicators in the model. Psychiatric indicators assessed diagnosis with schizophrenia (ICD-9 295), bipolar disorder (ICD-9 296.0-296.1, 296.4-296.8), depressive disorder (ICD-9 296.2-296.3, 300.4, 311), post-traumatic stress disorder (ICD-9 309.81), alcohol dependence (ICD-9 303, 305.0), and anxiety disorders (ICD-9 300.00, 300.02, 300.09), [15] to which was added an indicator of substance use disorder (ICD-9 291, 292, 303-305).

Outcomes were hospital admission events (any versus none). Psychiatric admissions were identified by a hospitalization with a primary diagnosis of a psychiatric disorder in the range reviewed above including those not specified (ICD-9 codes 290-311). Psychiatric diagnoses in the range ICD-9 312-319 are related to childhood/ mental retardation and are extremely rare in the postmilitary population that uses the VA. There were 25 persons with admissions attributed to this range of diagnosis codes; these individuals were excluded from the study, leaving a sample size of 824,483 persons. Medical admissions were all those with a primary diagnosis outside the range 290-319. Epilepsy-specific admissions, a subset of medical admissions, were admissions with a primary diagnosis of a seizure disorder (ICD-9 345, 780.39). We report these separately to establish that medical admissions of the new-onset epilepsy patients were not solely related to their epilepsy.

\section{Analysis}

After characterizing the sample with descriptive statistics, adjusted logistic regression models assessed factors in admission (yes/no) in FY2000, including demographic 
measures, the indicators of prior neurological conditions, clinical covariates (medical and psychiatric comorbidities) to adjust for between-group differences, and new-onset epilepsy. Separate models assessed each outcome: psychiatric admission and medical admission. A second set of models restricted to the subset of patients with new-onset epilepsy determined factors in hospitalization specific to TIGER patients. All models included an indicator of loss to follow-up, which identified patients for whom no data was found in FY2001. This included patients who died in FY2001, left the VA system, or remained hospitalized past the end of the observation period at the end of FY2001. In analysis, many modest effects achieve statistical significance when the sample is very large as in this study, therefore we focused on odds ratios indicating medium ( $\geq 1.5$ for positive associations and $\leq 0.67$ for inverse associations) or large ( $\geq 2$ or of $\leq .5$ ) effect sizes [17]. Model fit was reported by the c-statistic, a measure which ranges from 0.5 for model fit no better than by chance to 1.0 for perfect fit.

\section{Results \\ Sample}

The TIGER study of 824,483 patients aged 66 or older in FY2000 included 16,814 women (2\%). Consistent with U. S. military recruitment trends, most patients were Anglo males with 12\% African-American and 5\% Hispanic patients (Table 1). There were 1,610 patients with new-onset epilepsy (TIGER patients) in the sample (0.2\%). TIGER patients resembled non-epilepsy patients on most demographic measures (age, gender) but differed in that they were more likely to be African-American (20\% of TIGER patients versus $12 \%$ of non-TIGER patients, chi-square $=115.6, \mathrm{df}=2, \mathrm{p}<.0001)$. Summing the included and excluded patients for the denominator and adjusting for the deleted cases missing data on race, the incidence of epilepsy in late-life VA patients was 156 cases per 100,000 patients while prevalence was 42 per 1,000 .

\section{Comorbidity}

New-onset epilepsy patients had high rates of the prior neurological conditions studied: cerebrovascular disease $(40 \%$ TIGER versus $17 \%$ other VA patients over age 66$)$, dementia ( $18 \%$ versus $7 \%)$, brain tumor $(2 \%$ versus $1 \%)$ and head injury $(2 \%$ versus $<1 \%)$. Psychiatric comorbid conditions were also common, especially depressive disorders which were diagnosed in $17 \%$ of new-onset epilepsy patients compared to $9 \%$ of nonepilepsy patients. Overall, these older patients averaged $3.0( \pm 2.0)$ Selim physical comorbid conditions of 29 assessed conditions and $0.22( \pm 0.56)$ Selim mental illnesses of six assessed.

\section{Outcomes}

The proportion of geriatric patients with a hospital admission varied by new-onset epilepsy status. Overall, the proportion hospitalized was $15 \%(121,118)$ in FY00 but it was $52 \%$ (844) for the subset of new-onset epilepsy patients. Most patients had medical admissions $(14 \% ; n=116,357)$ while $1 \%(7,830)$ experienced psychiatric admissions; for TIGER patients these proportions were 50\% (809) and 6\% (91). Some patients had both types of admissions: $0.4 \%(3,069)$ of the large sample and $3.5 \%$ (56) of the new-onset epilepsy patients. Among TIGER patients, roughly one-third with medical admissions were admitted specifically for epilepsy as the primary diagnosis for the hospitalization (ICD9 codes 345.xx or 780.39). Only 19 TIGER patients had both psychiatric and epilepsy-specific admissions. The next year in FY01, $14 \%$ of all patients $(106,483)$ were admitted as were $29 \%$ of new-onset epilepsy patients (387). TIGER patients were more likely to be lost from the study (chi-square 59.4, d.f. $=2$, $\mathrm{p}<.0001$ ), primarily through death.

Days of hospitalization for medical admissions averaged 19.5 days ( \pm 29.8 ; median 9 days) for the entire sample, and were markedly higher for TIGER patients (median 14.0 vs 9.0 days; Wilcoxon 2 -sample $\mathrm{z}$-statistic $=10.4$; two-sided $\mathrm{p}<.0001)$. Individuals with new-onset epilepsy had somewhat more inpatient days for psychiatric reasons compared to other patients: mean 52.7 ( \pm 58.7 ) versus $40.8( \pm 47.3)$, and median 32 vs 24 days (Wilcoxon 2-sample z-statistic $=2.03$; two-sided $\mathrm{p}=$ $.04)$.

In the covariate-adjusted model, psychiatric hospital admission was primarily associated with psychiatric disorders for these geriatric patients but also showed strong associations with prior neurological conditions: cerebrovascular disease $(\mathrm{OR}=1.5,95 \% \mathrm{CI} 1.4-1.6)$, dementia $(\mathrm{OR}=9.5,95 \%$ CI 9.0-10.0), alcohol dependence $(\mathrm{OR}=11.7 ; 95 \% \mathrm{CI}$ 10.9-12.5), schizophrenia (OR = 6.3; $95 \%$ CI 5.9-6.7), bipolar disorder $(\mathrm{OR}=4.6 ; 95 \%$ CI 4.2-5.0), depression (OR $=2.8$; 95\% CI 2.7-3.0). Newonset epilepsy was also associated with increased risk of psychiatric admission ( $\mathrm{OR}=2.9,95 \% \mathrm{CI} 2.2-3.7)$. An inverse relationship with psychiatric admission was noted for Hispanic ethnicity $(\mathrm{OR}=0.58,95 \%$ CI 0.51 $0.65)$. Some physical conditions had modest associations, with hepatitis strongest among these $(\mathrm{OR}=1.5$; $95 \% \mathrm{CI}$ 1.3-1.7). Complete multivariable results are reported in Table 2.

In the model of medical admissions among 824,483 geriatric patients, significant factors were noted in each domain. The demographic factor of African American race was associated with greater risk of medical admission $(\mathrm{OR}=1.5$; 95\% CI 1.4-1.5). Among prior neurological conditions, new-onset epilepsy imparted 5-fold 
Table 1 Description of Elderly Patients in the Veterans Health Administration in FY2000

\begin{tabular}{|c|c|c|c|}
\hline Characteristic & TIGER Cohort with New-Onset Epilepsy & Non-Epilepsy Patients & Total Sample \\
\hline \multirow[t]{2}{*}{ Number of patients } & 1,610 & 822,873 & 824,483 \\
\hline & N (\%) or Mean (SD) & N (\%) or Mean (SD) & N (\%) or Mean (SD) \\
\hline \multicolumn{4}{|l|}{ Age } \\
\hline $65-74$ Years & $850(53 \%)$ & $448,274(54 \%)$ & $449,124(54 \%)$ \\
\hline 75-84 Years & 706 (44\%) & $345,985(42 \%)$ & $346,691(42 \%)$ \\
\hline 85 or older & $54(3 \%)$ & $28,614(3 \%)$ & $28,668(3 \%)$ \\
\hline \multicolumn{4}{|l|}{ Ethnicity/Race } \\
\hline Hispanic & $83(5 \%)$ & $44,612(5 \%)$ & $44,695(5 \%)$ \\
\hline African-American & $323(20 \%)$ & $94,669(12 \%)$ & $94,992(12 \%)$ \\
\hline Anglo & $1,204(75 \%)$ & $683,592(83 \%)$ & $684,796(83 \%)$ \\
\hline \multicolumn{4}{|l|}{ Gender } \\
\hline Women & $24(1.5 \%)$ & $16,790(2 \%)$ & $16,814(2 \%)$ \\
\hline Men & $1,586(98.5 \%)$ & $806,083(98 \%)$ & 807,669 (98\%) \\
\hline Neurological conditions, other & $150(9 \%)$ & $32,114(4 \%)$ & $32,264(4 \%)$ \\
\hline Cerebrovascular disease & $639(40 \%)$ & $136,306(17 \%)$ & $136,945(17 \%)$ \\
\hline Dementia & $284(18 \%)$ & $60,588(7 \%)$ & $60,872(7 \%)$ \\
\hline Brain tumor & $27(2 \%)$ & $5,306(1 \%)$ & $5,333(1 \%)$ \\
\hline Head injury & $25(2 \%)$ & $3,565(<1 \%)$ & $3,590(<1 \%)$ \\
\hline Any psychiatric condition & $801(50 \%)$ & $199,480(24 \%)$ & $200,281(24 \%)$ \\
\hline Anxiety & $152(9 \%)$ & $43,292(5 \%)$ & $43,444(5 \%)$ \\
\hline Depression & $280(17 \%)$ & $71,623(9 \%)$ & $71,903(9 \%)$ \\
\hline Post-traumatic stress disorder & $77(5 \%)$ & $22,382(3 \%)$ & $22,459(3 \%)$ \\
\hline Bipolar disorder & $47(3 \%)$ & $9,729(1 \%)$ & $9,776(1 \%)$ \\
\hline Schizophrenia & $80(5 \%)$ & $15,954(2 \%)$ & $16,034(2 \%)$ \\
\hline Alcohol dependence & $104(6 \%)$ & $16,922(2 \%)$ & $17,026(2 \%)$ \\
\hline Substance abuse & $211(13 \%)$ & $55,554(7 \%)$ & $55,765(7 \%)$ \\
\hline Hypertension & $1,147(71 \%)$ & $510,382(62 \%)$ & $511,529(62 \%)$ \\
\hline Dyslipidemia & 449 (28\%) & $265,785(32 \%)$ & $266,234(32 \%)$ \\
\hline Diabetes & 497 (31\%) & $221,810(27 \%)$ & $222,307(27 \%)$ \\
\hline FY00 psychiatric admission & $91(6 \%)$ & $7,739(1 \%)$ & $7,830(1 \%)$ \\
\hline FY00 medical admission & 809 (50\%) & $115,548(14 \%)$ & $116,357(14 \%)$ \\
\hline $\begin{array}{l}\text { FY00 epilepsy-related admission } \\
\text { (a subset of medical admissions) }\end{array}$ & $263(16 \%)$ & $4(<1 \%)$ & $267(<1 \%)$ \\
\hline
\end{tabular}

increased relative odds (OR $=4.8 ; 95 \%$ CI 4.3-5.5). Other neurologic risk factors roughly doubled relative odds of medical admission: dementia $(\mathrm{OR}=2.2$; $95 \%$ CI 2.1-2.3), head injury ( $\mathrm{OR}=2.2 ; 95 \%$ CI 2.0-2.4), brain tumor $(\mathrm{OR}=1.9 ; 95 \% \mathrm{CI} 1.8-2.1)$, cerebrovascular disease $(\mathrm{OR}=1.8 ; 95 \% \mathrm{CI} 1.7-1.8)$, and other neurological disorders such as multiple sclerosis and Parkinsons $(\mathrm{OR}=1.8$; 95\% CI 1.8-1.9). Many other physical ills were associated with medical admission, notably comorbid gall bladder disorders $(\mathrm{OR}=7.4$; 95\% CI 6.9-7.8), myocardial infarct $(\mathrm{OR}=4.7 ; 95 \% \mathrm{CI}$
4.5-4.8), angina ( $\mathrm{OR}=3.5 ; 95 \% \mathrm{CI} 3.4-3.6)$, and anemia $(\mathrm{OR}=3.2$; $95 \%$ CI 3.1-3.3). Among mental disorders, significant associations with medical admission were found for alcohol dependence $(\mathrm{OR}=2.3$; 95\% CI 2.22.4) and schizophrenia (OR $=1.5 ; 95 \%$ CI 1.4-1.6; Table 3).

For the subset of 1,610 new-onset epilepsy patients, the model of psychiatric admissions found an effect of prior dementia (OR $=2.3 ; 95 \%$ CI 1.4-4.0), and hypertension was a risk factor for psychiatric admission (OR $=2.2 ; 95 \%$ CI 1.2-4.1) while peripheral vascular disease 
Table 2 Factors Associated with Psychiatric Admissions among Geriatric Patients ( $N=824,483$; c-statistic $=0.95$ )

\begin{tabular}{|c|c|c|c|}
\hline Effect & $\begin{array}{l}\text { Odds } \\
\text { Ratio }\end{array}$ & $95 \% \mathrm{Cl}$ & $\begin{array}{c}\text { Significant } \\
\text { (Cl excludes 1.0) }\end{array}$ \\
\hline Alcohol dependence & 11.70 & $10.9-12.5$ & * \\
\hline Dementia & 9.50 & $9.00-10.0$ & * \\
\hline Schizophrenia & 6.29 & $5.87-6.73$ & * \\
\hline Bipolar disorder & 4.59 & $4.23-4.97$ & * \\
\hline New-onset epilepsy & 2.87 & $2.24-3.67$ & * \\
\hline Depression & 2.83 & $2.68-2.99$ & * \\
\hline Lost to follow-up & 2.01 & $1.89-2.14$ & * \\
\hline PTSD & 1.67 & $1.52-1.82$ & * \\
\hline Cerebrovascular disease & 1.50 & $1.42-1.59$ & * \\
\hline Hepatitis & 1.46 & $1.27-1.68$ & * \\
\hline $\begin{array}{l}\text { Neurological conditions, } \\
\text { other }\end{array}$ & 1.42 & $1.32-1.53$ & * \\
\hline Anemia & 1.40 & $1.32-1.50$ & * \\
\hline Anxiety & 1.40 & $1.30-1.50$ & * \\
\hline COPD & 1.38 & $1.30-1.45$ & * \\
\hline Peptic ulcer & 1.38 & $1.24-1.53$ & * \\
\hline Age 85 or older & 1.37 & $1.23-1.52$ & * \\
\hline Brain tumor & 1.30 & $1.03-1.65$ & * \\
\hline Irregular heartbeat & 1.24 & $1.17-1.33$ & * \\
\hline Heart attack & 1.23 & $1.12-1.36$ & * \\
\hline Female & 1.22 & $1.05-1.43$ & * \\
\hline Head injury & 1.22 & $1.00-1.49$ & * \\
\hline African American & 1.21 & $1.13-1.30$ & * \\
\hline Hypertension & 1.21 & $1.15-1.28$ & * \\
\hline Low-back pain & 1.18 & $1.10-1.27$ & * \\
\hline Other arthritic disease & 1.17 & $1.05-1.29$ & * \\
\hline Enlarged prostate & 1.16 & $1.10-1.23$ & * \\
\hline Hip problems & 1.11 & $0.96-1.27$ & n.s. \\
\hline Prostatitis & 1.10 & $0.90-1.35$ & n.s. \\
\hline Osteoarthritis & 1.07 & $1.00-1.13$ & * \\
\hline Gallbladder disease & 0.98 & $0.79-1.21$ & n.s. \\
\hline Gout & 0.98 & $0.87-1.11$ & n.s. \\
\hline Peripheral vascular disease & 0.97 & $0.89-1.05$ & n.s. \\
\hline Angina & 0.95 & $0.86-1.05$ & n.s. \\
\hline Diverticulitis & 0.95 & $0.84-1.08$ & n.s. \\
\hline Congestive Heart Failure & 0.94 & $0.87-1.01$ & n.s. \\
\hline Age $75-84$ years & 0.92 & $0.87-0.97$ & * \\
\hline Cancer & 0.84 & $0.79-0.90$ & * \\
\hline Rheumatoid arthritis & 0.84 & $0.68-1.03$ & \\
\hline Cataracts & 0.80 & $0.75-0.86$ & * \\
\hline Inflammatory bowel disease & 0.64 & $0.43-0.96$ & * \\
\hline Hispanic & 0.58 & $0.51-0.65$ & * \\
\hline
\end{tabular}

was inversely associated (OR $=0.36 ; 95 \%$ CI .14-.87). Age and race/ethnicity were not significantly related. Psychiatric diagnoses were related as expected, especially alcohol dependence $(\mathrm{OR}=5.2,95 \%$ CI 2.6-10.0) and schizophrenia $(\mathrm{OR}=3.5 ; 95 \%$ CI 1.7-7.3). Details are reported in Table 4.
Table 3 Factors Associated with Medical Admissions among Geriatric Patients ( $N=824,483$; c-statistic $=0.86$ )

\begin{tabular}{|c|c|c|c|}
\hline Effect & Odds Ratio & $95 \% \mathrm{Cl}$ & \\
\hline Gallbladder disease & 7.37 & $6.94-7.82$ & * \\
\hline New-onset epilepsy & 4.84 & $4.29-5.46$ & * \\
\hline Heart attack & 4.68 & $4.55-4.81$ & * \\
\hline Lost to follow-up & 3.74 & $3.67-3.82$ & * \\
\hline Angina & 3.51 & $3.42-3.60$ & * \\
\hline Anemia & 3.20 & $3.14-3.26$ & * \\
\hline Hip problems & 2.75 & $2.63-2.88$ & * \\
\hline Irregular heartbeat & 2.59 & $2.54-2.64$ & * \\
\hline Cancer & 2.45 & $2.41-2.49$ & * \\
\hline COPD & 2.41 & $2.37-2.45$ & * \\
\hline Hepatitis & 2.38 & $2.25-2.51$ & * \\
\hline Alcohol dependence & 2.30 & $2.21-2.40$ & * \\
\hline Congestive Heart Failure & 2.21 & $2.17-2.25$ & * \\
\hline Dementia & 2.20 & $2.15-2.25$ & * \\
\hline Head injury & 2.19 & $2.01-2.38$ & * \\
\hline Peptic ulcer & 1.97 & $1.91-2.04$ & * \\
\hline Brain tumor & 1.93 & $1.80-2.07$ & * \\
\hline Inflammatory bowel disease & 1.92 & $1.76-2.10$ & * \\
\hline Peripheral vascular disease & 1.83 & $1.79-1.87$ & * \\
\hline Neurological conditions, other & 1.81 & $1.75-1.87$ & * \\
\hline Cerebrovascular disease & 1.75 & $1.72-1.78$ & * \\
\hline Diverticulitis & 1.73 & $1.68-1.79$ & * \\
\hline Prostatitis & 1.55 & $1.46-1.64$ & * \\
\hline Hypertension & 1.48 & $1.45-1.50$ & * \\
\hline Schizophrenia & 1.48 & $1.41-1.55$ & * \\
\hline Rheumatoid arthritis & 1.47 & $1.40-1.54$ & * \\
\hline African American & 1.45 & $1.42-1.48$ & * \\
\hline Enlarged prostate & 1.40 & $1.37-1.42$ & * \\
\hline Female & 1.39 & $1.32-1.47$ & * \\
\hline Gout & 1.33 & $1.29-1.38$ & * \\
\hline Other arthritic disease & 1.30 & $1.26-1.34$ & * \\
\hline Low-back pain & 1.26 & $1.23-1.28$ & * \\
\hline Depression & 1.24 & $1.21-1.27$ & * \\
\hline Hispanic & 1.20 & $1.16-1.24$ & * \\
\hline Osteoarthritis & 1.14 & $1.12-1.16$ & * \\
\hline Anxiety & 1.12 & $1.09-1.16$ & * \\
\hline Age 85 or older & 1.11 & $1.07-1.16$ & * \\
\hline Cataracts & 1.10 & $1.08-1.12$ & * \\
\hline Bipolar disorder & 1.07 & $1.01-1.14$ & * \\
\hline Age $75-84$ years & 0.90 & $0.89-0.92$ & * \\
\hline PTSD & 0.82 & $0.79-0.86$ & * \\
\hline
\end{tabular}

The model of medical admissions among new-onset epilepsy patients found that both African American race $(\mathrm{OR}=2.3 ; 95 \% \mathrm{CI} 1.7-3.1)$ and Hispanic ethnicity (OR $=2.4$; $95 \%$ CI 1.4.4.2) were associated with greater relative odds of admission. The prior neurological factors of cerebrovascular disease $(\mathrm{OR}=1.7 ; 95 \% \mathrm{CI} 1.3-2.2)$ and dementia $(\mathrm{OR}=1.5$; 95\% CI 1.1-2.1) were significant. Among physical disorders, most strongly related were 
Table 4 Factors Associated with Psychiatric Admissions among Geriatric Patients with New-Onset Epilepsy (N $=1,610$; c-statistic $=0.83$ ).

\begin{tabular}{|c|c|c|c|}
\hline Effect & $\begin{array}{l}\text { Odds } \\
\text { Ratio }\end{array}$ & $95 \% \mathrm{Cl}$ & $\begin{array}{c}\text { Significant } \\
\text { (Cl excludes 1.0) }\end{array}$ \\
\hline Alcohol dependence & 5.17 & $2.66-10.1$ & * \\
\hline Schizophrenia & 3.38 & $1.63-7.01$ & * \\
\hline Bipolar disorder & 2.79 & $1.12-6.96$ & * \\
\hline Dementia & 2.24 & $1.32-3.81$ & * \\
\hline Hypertension & 2.14 & $1.16-4.05$ & * \\
\hline Depression & 2.14 & $1.27-3.62$ & * \\
\hline PTSD & 2.10 & $0.94-4.68$ & n.s. \\
\hline Hepatitis & 2.03 & $0.58-7.02$ & n.s. \\
\hline Anxiety & 1.81 & $0.96-3.41$ & n.s. \\
\hline Other arthritic disease & 1.75 & $0.80-3.83$ & n.s. \\
\hline Gout & 1.60 & $0.58-4.41$ & n.s. \\
\hline COPD & 1.50 & $0.91-2.49$ & n.s. \\
\hline Angina & 1.46 & $0.69-3.08$ & n.s. \\
\hline Ulcer peptic & 1.44 & $0.59-3.55$ & n.s. \\
\hline Low-back pain & 1.43 & $0.79-2.60$ & n.s. \\
\hline Irregular heartbeat & 1.39 & $0.79-2.49$ & n.s. \\
\hline $\begin{array}{l}\text { Cerebrovascular } \\
\text { disease }\end{array}$ & 1.37 & $0.83-2.26$ & n.s. \\
\hline Anemia & 1.33 & $0.76-2.32$ & n.s. \\
\hline Diverticulitis & 1.32 & $0.49-3.61$ & n.s. \\
\hline Age $75-84$ years & 1.27 & $0.77-2.09$ & n.s. \\
\hline $\begin{array}{l}\text { Neurological } \\
\text { conditions, other }\end{array}$ & 1.27 & $0.63-2.59$ & n.s. \\
\hline Hip problems & 1.27 & $0.42-3.79$ & n.s. \\
\hline Rheumatoid arthritis & 1.27 & $0.25-6.38$ & n.s. \\
\hline Heart attack & 1.26 & $0.61-2.87$ & n.s. \\
\hline Lost to follow-up & 1.24 & $0.65-2.37$ & n.s. \\
\hline African American & 1.22 & $0.67-2.20$ & n.s. \\
\hline Enlarged prostate & 1.18 & $0.70-2.01$ & n.s. \\
\hline Cataracts & 0.99 & $0.54-1.79$ & n.s. \\
\hline $\mathrm{CHF}$ & 0.99 & $0.53-1.86$ & n.s. \\
\hline Hispanic & 0.98 & $0.34-2.79$ & n.s. \\
\hline Thyroid & 0.81 & $0.34-1.93$ & n.s. \\
\hline Gallbladder disease & 0.80 & $0.14-4.55$ & n.s. \\
\hline Osteoarthritis & 0.77 & $0.42-1.39$ & n.s. \\
\hline Diabetes & 0.76 & $0.44-1.29$ & n.s. \\
\hline Cancer & 0.64 & $0.34-1.19$ & n.s. \\
\hline Prostatitis & 0.60 & $0.05-7.16$ & n.s. \\
\hline Age 85 or older & 0.53 & $0.07-4.19$ & n.s. \\
\hline Brain tumor & 0.43 & $0.05-4.09$ & n.s. \\
\hline Head injury & 0.35 & $0.04-2.89$ & n.s. \\
\hline $\begin{array}{l}\text { Peripheral vascular } \\
\text { disease }\end{array}$ & 0.34 & $0.14-0.84$ & * \\
\hline
\end{tabular}

myocardial infarction $(\mathrm{OR}=4.7 ; 95 \% \mathrm{CI} 2.7-8.3)$ and arrhythmias $(\mathrm{OR}=2.3$; 95\% CI 1.7-3.1). Finally, the mental disorder alcohol dependence was again correlated with medical admission $(\mathrm{OR}=2.5$; 95\% CI 1.5-4.1; Table 5).
Table 5 Factors Associated with Medical Admissions among Geriatric Patients with New-Onset Epilepsy ( $N=1,610$; c-statistics $=0.80$ ).

\begin{tabular}{|c|c|c|c|}
\hline Effect & $\begin{array}{l}\text { Odds } \\
\text { Ratio }\end{array}$ & $95 \% \mathrm{Cl}$ & $\begin{array}{c}\text { Significant } \\
\text { (Cl excludes 1.0) }\end{array}$ \\
\hline Heart attack & 4.74 & $2.72-8.28$ & * \\
\hline Gallbladder disease & 3.90 & $1.21-12.58$ & * \\
\hline Anemia & 2.93 & $2.09-4.10$ & * \\
\hline Angina & 2.57 & $1.57-4.22$ & * \\
\hline Alcohol dependence & 2.46 & $1.49-4.08$ & * \\
\hline Head injury & 2.44 & $0.88-6.81$ & n.s. \\
\hline Hispanic & 2.35 & $1.35-4.09$ & * \\
\hline Brain tumor & 2.30 & $0.74-7.14$ & n.s. \\
\hline Irregular heartbeat & 2.26 & $1.66-3.08$ & * \\
\hline African American & 2.25 & $1.67-3.05$ & * \\
\hline Lost to follow-up & 2.12 & $1.53-2.94$ & * \\
\hline Cancer & 2.08 & $1.56-2.78$ & * \\
\hline Hip problems & 2.05 & $0.98-4.27$ & n.s. \\
\hline Thyroid & 1.87 & $1.15-3.03$ & * \\
\hline Ulcer peptic & 1.73 & $0.94-3.16$ & n.s. \\
\hline $\begin{array}{l}\text { Cerebrovascular } \\
\text { disease }\end{array}$ & 1.72 & $1.34-2.19$ & * \\
\hline COPD & 1.66 & $1.27-2.18$ & * \\
\hline Schizophrenia & 1.65 & $0.93-2.90$ & n.s. \\
\hline $\begin{array}{l}\text { Peripheral vascular } \\
\text { disease }\end{array}$ & 1.61 & $1.11-2.34$ & * \\
\hline Dementia & 1.54 & $1.12-2.12$ & * \\
\hline Enlarged prostate & 1.53 & $1.16-2.02$ & * \\
\hline Hypertension & 1.51 & $1.15-1.98$ & $*$ \\
\hline Diabetes & 1.46 & $1.13-1.89$ & * \\
\hline Rheumatoid arthritis & 1.46 & $0.59-3.64$ & n.s. \\
\hline $\mathrm{CHF}$ & 1.44 & $1.02-2.05$ & * \\
\hline $\begin{array}{l}\text { Neurological } \\
\text { conditions, other }\end{array}$ & 1.39 & $0.92-2.10$ & n.s. \\
\hline Anxiety & 1.29 & $0.84-1.98$ & n.s. \\
\hline Prostatitis & 1.27 & $0.41-3.93$ & n.s. \\
\hline Other arthritic disease & 1.22 & $0.75-1.99$ & n.s. \\
\hline Osteoarthritis & 1.16 & $0.86-1.56$ & n.s. \\
\hline Gout & 1.11 & $0.59-2.07$ & n.s. \\
\hline Bipolar disorder & 1.11 & $0.55-2.25$ & n.s. \\
\hline Cataracts & 1.09 & $0.81-1.47$ & n.s. \\
\hline PTSD & 1.07 & $0.60-1.91$ & n.s. \\
\hline Depression & 1.00 & $0.72-1.39$ & n.s. \\
\hline Hepatitis & 0.98 & $0.37-2.62$ & n.s. \\
\hline Age 85 or older & 0.90 & $0.46-1.78$ & n.s. \\
\hline Low-back pain & 0.89 & $0.63-1.25$ & n.s. \\
\hline Diverticulitis & 0.84 & $0.46-1.53$ & n.s. \\
\hline Age $75-84$ years & 0.81 & $0.64-1.04$ & n.s. \\
\hline
\end{tabular}

\section{Discussion}

To our knowledge, this is the first paper to examine the high rates of hospital admissions among elderly newonset epilepsy patients (52\%), relative to elderly patients without epilepsy (15\%). Results suggest that the 
diagnosis of epilepsy in this population signifies a risk not only for recurrent seizures but also for hospitalization for a diversity of medical and psychiatric conditions. In this large sample of older patients using the VA healthcare system, those with new-onset epilepsy had more admissions compared to similar patients without epilepsy. Our study also highlights the association of multiple comorbidities and race/ethnicity with hospitalization among older veterans, and, strikingly, of alcohol dependence (see Figure 1). Alcohol dependence in the elderly is under-diagnosed yet a major factor in hospitalizations in this age group [18].

Echoing prior population-based studies, TIGER patients with new-onset epilepsy showed more than doubled rates of cerebrovascular disease, dementia, and psychiatric disorders - conditions associated with or sharing etiology with epilepsy. Future studies could examine which medical conditions present at the onset of epilepsy may contribute more to the increase in admissions, and how these affect a patient's course of treatment. In this elderly sample, epilepsy imparted an increased risk of both medical and psychiatric admission by a factor of 3 and 6 , respectively.

The incidence of epilepsy noted among these VA patients aged 66 years or older (156 cases/100,000 patients) is well above the range in population-based studies reviewed by Banerjee and colleagues, 16-51 per 100,000 [3]. The high incidence in this VA sample may be attributable to their elderly age, patient status, and to the generally high comorbidity seen in the VA patient population [19]. The VA preferentially provides care to veterans who are sicker, more disabled, impoverished, or combat-exposed.

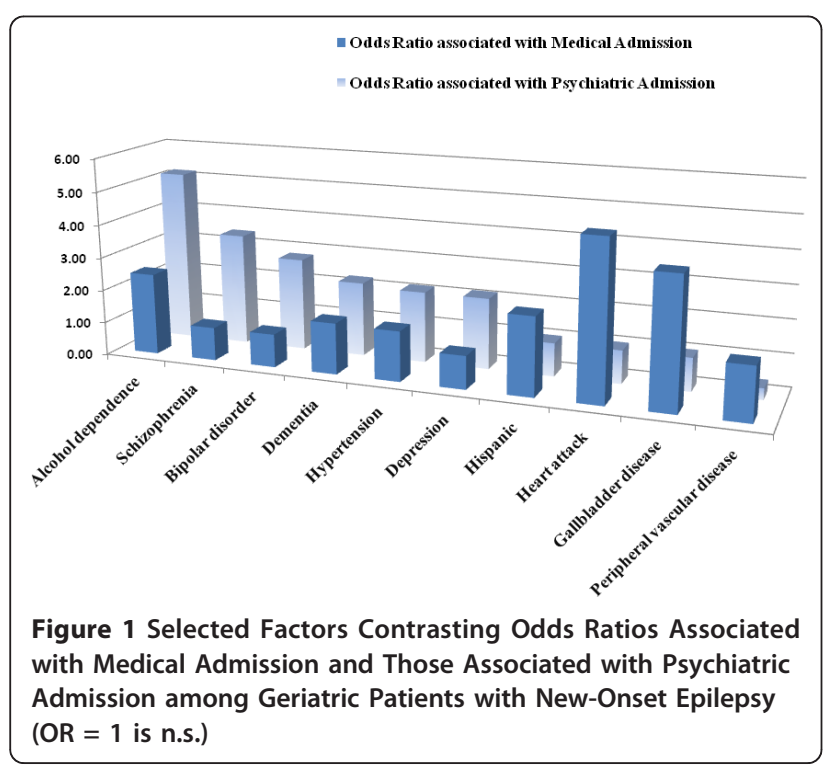

The findings regarding race/ethnicity are of note. Unexpectedly, minority race/ethnicity patients with new-onset epilepsy were at higher risk for hospitalization for medical reasons compared to patients not identified as white. At the same time, Hispanic ethnicity was associated with less risk of psychiatric admission in the total-sample analysis (but not in the new-onset epilepsy sub-analysis). In other words, non-white patients had relatively higher risk of medical admission, and the effect was stronger for non-white patients with newonset epilepsy. The Selim physical comorbidity scores for Hispanic patients $(2.8+/-1.9)$ were marginally lower than those of white patients $(2.9+/-2.0)$; African-American patients had slightly higher scores $(3.3+/-2.1)$ and were also more likely to have new-onset epilepsy. Whether non-white patients have less physiological reserve to cope with new-onset epilepsy, or differences in the specific comorbid conditions account for admission differences, is unclear.

TIGER patients hospitalized with a primary diagnosis of psychiatric disorder, epilepsy, or non-epilepsy medical conditions had notable differences in neurological and psychiatric comorbidity. A new diagnosis of epilepsy in a geriatric patient should not be viewed as an event easily dismissed with the prescription of an anti-epileptic drug. Rather the clinician should view this development as one attended by high risk of hospitalization and associated with a complex care plan. Furthermore, this may be a signal to consider caretaker availability, health education needs, and potentially end-of-life planning. In addition, limitations in patient-provider communication often arise for mental health patients, such that recognition of medical needs may be impeded in psychiatric patients. Clinicians who are not mental health specialists may want to consult with a geriatric psychiatrist to optimize a coordinated treatment plan, while mental health professionals will want to establish cooperative relationships with the medical specialists.

Comorbidity in epilepsy patients has been studied in only a few large studies, notably the Canadian and United Kingdom studies already cited, where high rates of digestive system disorders, stroke, respiratory disorders, dementia, migraine and other pain conditions as well as doubled co-occurring psychiatric disorders were noted $[2,4]$. VA patients are generally sicker than US residents [19]. The "protective" effect of peripheral vascular disease, which was inversely associated with psychiatric hospitalization among new-onset epilepsy, and the few other inversely related associations noted in the geriatric population (tables 2 and 3), merit further research but may be artifacts of the analysis, which estimated effects for a very large number of conditions simultaneously.

Limitations of this study include reliance on administrative data, gathered for purposes other than the 
current study and lacking in many measures that might be of interest, such as severity of illness scales and subjective experience reports. An additional limitation is uncertainty regarding the onset of epilepsy, as a person may have a seizure disorder for some time before it is recognized by diagnosis and treatment. Finally, the geriatric VA patient population is primarily male, reflecting military recruitment patterns, and VA patients are less healthy and wealthy than US residents in general [19], thus these results may not generalize to healthier patients nor to women.

\section{Conclusion}

Our findings highlight the role psychiatric and physical conditions play in both medical and psychiatric hospitalizations. Regardless of the type of hospitalization, substance abuse, dementia and cerebrovascular disease were strong risk factors for admission. Recognition of medical and mental health comorbidities is essential for appropriate treatment on both an inpatient and outpatient basis, and research is needed to determine whether race/ethnicity may play a role in the likelihood of admission type, considering that there may be underlying medical or equity issues to disentangle.

In assessing the inpatient care required by elderly VA patients, our examination of medical and psychiatric admissions found both considerable overlap between groups characterized by psychiatric and neurological conditions, and a large impact on inpatient utilization of epilepsy. From the patient point of view, having epilepsy appeared to increase the risk of medical admission as well as of psychiatric admission. From an analytic perspective, omitting epilepsy and other neurological conditions may lead to overestimation of the risk of admission attributable solely to psychiatric or common medical conditions. Finally, evaluated from a health systems, policy and resource allocation perspective, the emerging picture of the psychiatric epilepsy patient, characterized by a great need for healthcare resources, may merit development of practice guidelines to improve coordinated outpatient care and reduce demand on the healthcare system. As the Baby Boomer cohort ages into late life over the next two decades, this patient population and its healthcare needs are likely to expand greatly.

\section{Acknowledgements}

This research was supported by the Department of Veterans Affairs (Grant \#IIR-02-274; PI: Pugh) with additional support from the Center for Applied Health Research, Central Texas Veterans Health Care System, Scott \& White Healthcare System, VERDICT Research Center, and the University of Texas Health Science Center at San Antonio. The views expressed in this article are those of the authors and do not necessarily represent the views of the Department of Veterans Affairs.

\section{Author details}

${ }^{1}$ Veterans Affairs: Central Texas Veterans Health Care System (CAHR), 2102 Birdcreek Dr, Temple, TX 76502 USA. ${ }^{2}$ Scott \& White Healthcare, Center for Applied Health Research, Temple, TX USA. ${ }^{3}$ Neurological Surgery, P.C., Lake Success, NY USA. ${ }^{4}$ University of Texas Health Science Center at San Antonio, Department of Psychiatry, San Antonio, TX USA. ${ }^{5}$ Veterans Affairs HSR\&D: South Texas Veterans Health Care System (VERDICT), 7400 Merton Minter (11c6), San Antonio, TX 78229-4404 USA. ${ }^{6}$ University of Texas Health Science Center at San Antonio, Department of Epidemiology \& Biostatistics, San Antonio, TX USA.

\section{Authors' contributions}

LAC conceptualized the study, conducted the analyses, drafted and finalized the manuscript. JEZ contributed to the conceptual framework and writing. ABE provided clinical insight on issues regarding epilepsy and wrote portions of the final manuscript. JMG provided clinical insight on issues regarding psychiatric disorders and wrote portions of the final manuscript. MJP conceived, obtained funding for, and conducted the overall study, and contributed to interpreting study results, writing and finalizing the manuscript. All authors read and approved the final manuscript.

\section{Competing interests}

The authors declare that they have no competing interests.

Received: 7 July 2010 Accepted: 19 April 2011 Published: 19 April 2011

\section{References}

1. Hauser WA: Seizure disorders: the changes with age. Epilepsia 1992, 33: S6-14, PMID: 1425495.

2. Gaitatzis A, Carroll K, Majeed A, W Sander J: The epidemiology of the comorbidity of epilepsy in the general population. Epilepsia 2004, 45(12):1613-1622, PMID:15571520.

3. Banerjee PN, Filippi D, Allen Hauser W: The descriptive epidemiology of epilepsy-a review. Epilepsy Research 2009, 85(1):31-45, PMID 19369037.

4. Téllez-Zenteno JF, Matijevic S, Wiebe S: Somatic comorbidity of epilepsy in the general population in Canada. Epilepsia 2005, 46(12):1955-1962, PMID:16393162.

5. Pugh MJ, Knoefel JE, Mortensen EM, Amuan ME, Berlowitz DR, Van Cott AC: New-onset epilepsy risk factors in older veterans. Journal of the American Geriatrics Society 2009, 57(2):237-242, PMID: 19207140.

6. Annegers JF, Rocca WA, Hauser WA: Causes of epilepsy: contributions of the Rochester epidemiology project. Mayo Clinic Proceedings 1996, 71(6):570-575, PMID: 8642886

7. Pugh MJ, Zeber JE, Copeland LA, Tabares JV, Cramer JA: Psychiatric disease burden profiles among veterans with epilepsy: the association with health services utilization. Psychiatric Services 2008, 59(8):925-928, PMID: 18678692.

8. Ettinger AB, Copeland LA, Zeber JE, Van Cott A, Pugh MJ: Are psychiatric disorders independent risk factors for new-onset epilepsy in older individuals? Epilepsy \& Behavior 2010, 17(1):70-74, PMID: 19913462.

9. Linehan C, Kerr MP, Walsh PN, Brady G, Kelleher C, Delanty N, Dawson F, Glynn M: Examining the prevalence of epilepsy and delivery of epilepsy care in Ireland. Epilepsia 51(5):845-852, PMID: 20002142.

10. Faught RE, Weiner JR, Guerin A, Cunnington MC, Duh MS: Impact of nonadherence to antiepileptic drugs on health care utilization and costs: findings from the RANSOM study. Epilepsia 2009, 50(3):501-509, PMID: 19183224.

11. Henneman PL, DeRoos F, Lewis RJ: Determining the need for admission in patients with new-onset seizures. Annals of Emergency Medicine 1994, 24(6):1108-1114, PMID: 7978592.

12. Pugh MJ, Van Cott AC, Cramer JA, Knoefel JE, Amuan ME, Tabares J, Ramsay RE, Berlowitz DR: Trends in antiepileptic drug prescribing for older patients with new-onset epilepsy: 2000-2004. Neurology 2008, 70(22 Pt 2):2171-2178, PMID: 18505996.

13. Krishnan LL, Petersen NJ, Snow AL, Cully JA, Schulz PE, Graham DP, Morgan RO, Braun U, Moffett ML, Yu HJ, Kunik ME: Prevalence of dementia among Veterans Affairs medical care system users. Dementia and Geriatric Cognitive Disorders 2005, 20(4):245-253, PMID: 16088141.

14. Elixhauser A, Steiner C, Harris DR, Coffey RM: Comorbidity measures for use with administrative data. Medical Care 1998, 36(1):8-27, PMID: 9431328. 
15. Selim AJ, Fincke G, Ren XS, Lee A, Rogers WH, Miller DR, Skinner KM, Linzer M, Kazis LE: Comorbidity assessments based on patient report: results from the Veterans Health Study. The Journal of Ambulatory Care Management 2004, 27(3):281-295, PMID: 15287217.

16. Pugh MJ, Copeland LA, Zeber JE, Cramer JA, Amuan ME, Cavazos JE, Kazis LE: The impact of epilepsy on health status among younger and older adults. Epilepsia 2005, 46(11):1820-1827, PMID: 16302863.

17. Wickens TD: Multiway Contingency Tables Analysis for the Social Sciences Hillsdale NJ: Erlbaum; 1989

18. Adams WL, Yuan Z, Barboriak JJ, Rimm AA: Alcohol-related hospitalizations of elderly people. Prevalence and geographic variation in the United States. JAMA 1993, 270(10):1222-1225, PMID: 8355385.

19. Morgan RO, Teal CR, Reddy SG, Ford ME, Ashton CM: Measurement in Veterans Affairs Health Services Research: veterans as a special population. Health Services Research 2005, 40(5 Pt 2):1573-1583, PMID: 16178996.

\section{Pre-publication history}

The pre-publication history for this paper can be accessed here: http://www.biomedcentral.com/1472-6963/11/84/prepub

doi:10.1186/1472-6963-11-84

Cite this article as: Copeland et al:: Psychiatric and medical admissions observed among elderly patients with new-onset epilepsy. BMC Health Services Research 2011 11:84.

\section{Submit your next manuscript to BioMed Central and take full advantage of:}

- Convenient online submission

- Thorough peer review

- No space constraints or color figure charges

- Immediate publication on acceptance

- Inclusion in PubMed, CAS, Scopus and Google Scholar

- Research which is freely available for redistribution

Submit your manuscript at www.biomedcentral.com/submit 